\title{
COMMUTING TRACES OF BIADDITIVE MAPPINGS, COMMUTATIVITY-PRESERVING MAPPINGS AND LIE MAPPINGS
}

\author{
MATEJ BREŠAR
}

\begin{abstract}
Biadditive mappings $B: R \times R \rightarrow R$ where $R$ is a prime ring with certain additional properties, satisfying $B(x, x) x=x B(x, x)$ for all $x \in R$, are characterized. As an application we determine the structures of commutativity-preserving mappings, Lie isomorphisms, and Lie derivations of certain prime rings.
\end{abstract}

\section{INTRODUCTION, NOTATION, AND STATEMENTS OF THE RESULTS}

Throughout, $R$ and $R^{\prime}$ will represent associative rings. The center of $R$ and $R^{\prime}$ will be denoted by $Z$ and $Z^{\prime}$, respectively. We first recall a few facts concerning prime rings. Let $R$ be a prime ring (i.e., $a R b=0$ implies $a=0$ or $b=0)$. We will sketch the construction of Martindale's extended centroid of $R$ (see [23] and [17] for details). Define an equivalence relation on the set of all pairs $(U, f)$, where $U$ is a nonzero ideal of $R$ and $f: U \rightarrow R$ is a right $R$-module mapping of $U$ into $R$, by $(U, f) \sim(V, g)$ if $f=g$ on some nonzero ideal $W \subseteq U \cap V$. The set $Q$ of all equivalence classes forms a ring under the operations induced by addition and composition of representatives of the equivalence classes. $R$ embeds in $Q$ as left multiplication on $R$. The center $C$ of $Q$ is a field containing the centroid of $R . C$ is called the extended centroid of $R$. The $C$-algebra $R C+C$ is called the central closure of $R$. A prime algebra $A$ over a field $F$ is said to be a centrally closed prime algebra over $F$ if the center of $A$ is $F 1$, where 1 is the unit element of $A$, and $A$ is its own central closure, i.e., the extended centroid of $A$ is just $F 1$. The central closure of any prime ring is a centrally closed prime algebra over the extended centroid.

Throughout, by $C$ and $C^{\prime}$ we denote the extended centroid of a prime ring $R$ and $R^{\prime}$, respectively.

A mapping $f$ of $R$ into itself is called commuting if $[f(x), x]=0$ for all $x \in R$, where $[u, v]$ denotes the commutator $u v-v u$. In [34] Posner initiated the study of commuting and related mappings. He proved that if a prime ring $R$ admits a nonzero derivation $d$ satisfying $d[(x), x] \in Z$ for all $x \in R$ (such

Received by the editors May 1, 1990 and, in revised form, September 4, 1990.

1980 Mathematics Subject Classification (1985 Revision). Primary 16A72; Secondary 16A12, 16A68, 16A70.

Key words and phrases. Commuting mapping, trace of a biadditive mapping, commutativitypreserving mapping, Lie isomorphism, Lie triple isomorphism, Lie derivation, Lie triple derivation. 
mappings are called centralizing), then $R$ is commutative. Over the last twenty years a lot of work has been done on some additive commuting and centralizing mappings, especially derivations and endomorphisms; we refer the reader to some recent papers $[2,3,5,6,7,9,10,20,36]$ where further references can be found. In our forthcoming paper [6] the description of all additive commuting mappings of a prime ring $R$ is given. It is shown that every such mapping $f$ is of the form $f(x)=\lambda x+\zeta(x)$ where $\lambda$ is an element in $C$ and $\zeta$ is an additive mapping from $R$ into $C$. The analogous result was obtained for von Neumann algebras [5].

It seems that the first result on commuting mappings which are not additive was given by Vukman [36]. He proved that if $d$ is a derivation of a prime ring $R$ of characteristic not 2, such that the mapping $q(x)=[d(x), x]$ is commuting, then $q=0$ (that is, $d$ is commuting). In [7] we generalized this result by showing that the same conclusion holds for any additive mapping. In this paper we will, using methods similar to those in [7], describe all commuting traces of biadditive mappings on certain prime rings. By a trace of a biadditive mapping on a ring $R$ we mean a mapping $q: R \rightarrow R$ such that $q(x)=B(x, x)$, $x \in R$, for some biadditive mapping $B: R \times R \rightarrow R$. We will prove

Theorem 1. Let $R$ be a prime ring of characteristic not 2, and let $q: R \rightarrow R$ be a trace of a biadditive mapping. Suppose that $q$ is commuting. If $R$ does not satisfy $S_{4}$ then $q$ is of the form

$$
q(x)=\lambda x^{2}+\mu(x) x+\nu(x) \quad \text { for all } x \in R,
$$

where $\lambda$ is an element in $C, \mu$ is an additive mapping of $R$ into $C$, and $\nu$ is a mapping of $R$ into $C$.

Of course, Theorem 1 is a continuation of some our results mentioned above $[6,7]$. However, our main motivations for the study of commuting traces of biadditive mappings were the problems concerning commutativity-preserving mappings, Lie isomorphisms, and Lie derivations.

By a commutativity-preserving mapping we mean a mapping $\theta: R \rightarrow R^{\prime}$ satisfying $[x, y]=0$ implies $[\theta(x), \theta(y)]=0$. The obvious examples are mappings of the form

$$
\theta(x)=c \varphi(x)+f(x), \quad x \in R,
$$

where $c \in Z^{\prime}, \varphi$ is an isomorphism or an anti-isomorphism of $R$ into $R^{\prime}$, and $f$ is a mapping from $R$ into $Z^{\prime}$. The goal is to show that in certain cases these are in fact the only examples. It seems that the first result in this direction was given by Watkins [37] for the case where $R=R^{\prime}$ is the algebra of $n \times n$ matrices, $n \geq 4$, over a field, and $\theta$ is a bijective linear operator. Also, a simple counterexample was constructed for $n=2$. Subsequently, in the series of papers $[1,11,12,33,35]$ on commutativity-preserving mappings of matrices, the authors have refined Watkins's result in several ways; in particular, it has turned out that the result is also true for $n=3$, and that the similar conclusion holds for linear operators which preserve commuting pairs of symmetric matrices. The paper [12] of Choi, Jafarian, and Radjavi also contains some extensions of these results to the algebra of bounded linear operators on an infinite-dimensional Hilbert space. Further, Omladič [32] proved that if 
$L(X)$ is the algebra of all bounded linear operators on a nontrivial complex Banach space $X$, then every bijective linear operator $\theta: L(X) \rightarrow L(X)$ preserving commutativity in both directions (i.e., $x$ and $y$ commute if and only if $\theta(x)$ and $\theta(y)$ commute) is of the form (1). In [31] Miers obtained the analogous result for bijective, ${ }^{*}$-linear operators of von Neumann factors (i.e., prime von Neumann algebras).

We will study commutativity-preserving mappings in rather general algebras. In fact, we will consider the mappings $\theta$ satisfying the weaker assumption that for every element $x$ the elements $\theta(x)$ and $\theta\left(x^{2}\right)$ commute. There is a simple connection between such mappings and commuting traces of biadditive mappings. Indeed, let $\theta: R \rightarrow R^{\prime}$ be a bijective additive mapping satisfying $\left[\theta\left(x^{2}\right), \theta(x)\right]=0$ for all $x \in R$; that is, $\left[\theta\left(\theta^{-1}(\theta(x))^{2}\right), \theta(x)\right]=0$, and therefore,

$$
\left[\theta\left(\theta^{-1}(y)^{2}\right), y\right]=0 \text { for all } y \in R^{\prime} .
$$

That is, the mapping $y \mapsto \theta\left(\theta^{-1}(y)^{2}\right)$, which is a trace of the biadditive mapping $(y, z) \mapsto \theta\left(\theta^{-1}(y) \theta^{-1}(z)\right)$, is commuting.

Applying Theorem 1 we will be able to prove

Theorem 2. Let $A$ and $A^{\prime}$ be centrally closed prime algebras over a field $F$. Suppose that the characteristic of $A^{\prime}$ is different from 2, and suppose that $F \neq$ $G F(3)$. Let $\theta: A \rightarrow A^{\prime}$ be a bijective linear mapping satisfying $\left[\theta\left(x^{2}\right), \theta(x)\right]=$ 0 for all $x \in A$. If neither $A$ nor $A^{\prime}$ satisfies $S_{4}$ then

$$
\theta(x)=c \varphi(x)+f(x) \text { for all } x \in A,
$$

where $c \in F, c \neq 0, \varphi$ is an isomorphism or an anti-isomorphism of $A$ onto $A^{\prime}$, and $f$ is a linear mapping from $A$ into the center of $A^{\prime}$.

Mathieu [27] introduced the notion of an ultraprime normed algebra: a complex normed algebra $A$ is called ultraprime if there exists a constant $\alpha>0$ such that for any $a, b \in A$, the norm of the operator $x \mapsto a x b$ is at least $\alpha\|a\|\|b\|$. Every ultraprime algebra is, of course, prime, while the converse is not true. However, there are many important examples of ultraprime normed algebras, e.g., finite-dimensional prime normed algebras, prime $C^{*}$-algebras, and subalgebras of $L(X)$ that contain all finite rank operators. Every ultraprime algebra with unit element is centrally closed over the complex numbers. Thus Theorem 2 generalizes Miers' result [31] from prime von Neumann algebras to general prime $C^{*}$-algebras. Also, Theorem 2 implies that Omladič's result [32] holds for all subalgebras of $L(X)$ which contain the identity and all finite rank operators. Besides, in both cases the assumption that the mapping $\theta$ preserves the commutativity in both directions can be replaced by the weaker assumption that $\theta(x)$ and $\theta\left(x^{2}\right)$ commute for every $x$.

A Lie isomorphism of a ring $R$ onto a ring $R^{\prime}$ is a bijective additive mapping $\theta: R \rightarrow R^{\prime}$ which preserves commutators, i.e.,

$$
\theta([x, y])=[\theta(x), \theta(y)] \text { for all } x, y \in R .
$$

In [18] Hua showed that every Lie automorphisms of the ring $R$ of all $n \times n$ matrices over a division ring, $n \geq 3$, is of the form $\varphi+\tau$, where $\varphi$ is either an automorphism or a negative of an antiautomorphism of $R$ and $\tau$ is an additive mapping of $R$ into $Z$ sending commutators to zero. Somewhat later, 
in the series of papers [21, 24, 25] Martindale has extended Hua's theorem to more general rings. The deepest result in this series [25, Theorem 11] states that if $R$ is a prime ring with unit element 1 , of characteristic different from 2 and 3 and containing two nonzero idempotents whose sum is 1 , then every Lie isomorphism of $R$ onto a prime ring $R^{\prime}$ with unit element is of the form $\varphi+\tau$, where $\varphi$ is a homomorphism or a negative of an antihomomorphism of $R$ into the central closure of $R^{\prime}$ and $\tau$ is an additive map of $R$ into $C^{\prime}$ sending commutators to zero. The analogous result for von Neumann factors was obtained by Miers [28].

Obviously, every Lie isomorphism preserves commutativity. Thus Theorem 2 could be applied. However, it turns out that in the case of Lie isomorphisms some assumptions in Theorem 2 can be removed. Using the same basic ideas as in the proof of Theorem 2 we will obtain

Theorem 3. Let $R$ and $R^{\prime}$ be prime rings such that the characteristic of $R^{\prime}$ is not 2. Let $\theta: R \rightarrow R^{\prime}$ be a Lie isomorphism. If neither $R$ nor $R^{\prime}$ satisfies $S_{4}$ then $\theta$ is of the form $\varphi+\tau$, where $\varphi$ is a homomorphism or a negative of an antihomomorphism of $R$ into the central closure of $R^{\prime}, \varphi$ is one-to-one, and $\tau$ is an additive mapping of $R$ into $C^{\prime}$ sending commutators to zero.

An example given in [21] shows that the image of $\varphi$ need not be contained in $R^{\prime}$.

Martindale [25] posed the question whether the assumption of idempotents, which is required in all his theorems on Lie isomorphisms is necessary or not (see also the discussion on Lie and Jordan mappings in [26]). Thus Theorem 3 gives a partial answer to this question. We remark that in the study of commutativity-preserving mappings the arguments employed by some authors (e.g., in $[12,31,32])$ also rest heavily on the presence of idempotents.

Suppose that a centrally closed prime algebra $A$ over a field $F$ satisfies $S_{4}$. Then, for any $a \in A$, we have $a^{2}=\alpha_{1} a+\alpha_{2} 1$ for some $\alpha_{1}, \alpha_{2} \in F$ (cf. Lemma 1 below). Therefore, every linear mapping $\theta$ of $A$ that preserves the center of $A$ satisfies $\left[\theta\left(x^{2}\right), \theta(x)\right]=0$ for all $x \in A$. Thus Theorem 2 is definitive of its kind. On the contrary, it seems that Theorem 3 still needs some refinement. We leave as an open question whether or not in Theorem 3, as well as in some other results in this paper, the assumption that the rings do not satisfy $S_{4}$ can be removed.

A bijective additive mapping $\theta: R \rightarrow R^{\prime}$ is called a Lie triple isomorphism if it satisfies

$$
\theta([[x, y], z])=[[\theta(x), \theta(y)], \theta(z)] \text { for all } x, y, z \in R .
$$

The notion of a Lie triple isomorphism can be viewed as a common generalization of both the notion of a Lie isomorphism and a Jordan triple isomorphism (i.e., bijective mapping $\theta: R \rightarrow R^{\prime}$ satisfying $\theta(x y x)=\theta(x) \theta(y) \theta(x)$ (cf. [4 and 15])). In [19 and 29] the authors have showed that in certain rings every Lie triple homomorphism is either a Lie homomorphism or a Lie antihomomorphism. The methods in this paper enable a more direct approach. As a consequence of Theorem 2 and some of our results in [7] we will obtain

Corollary 1. Let $A$ and $A^{\prime}$ be centrally closed prime algebras over a field $F$. Suppose that the characteristic of $A^{\prime}$ is different from 2 and 3. Let $\theta: A \rightarrow A^{\prime}$ 
be a linear Lie triple isomorphism. If neither $A$ nor $A^{\prime}$ satisfies $S_{4}$ then $\theta$ is of the form

$$
\theta(x)= \pm \varphi(x)+f(x) \quad \text { for all } x \in A,
$$

where $\varphi$ is an isomorphism or an anti-isomorphism of $A$ onto $A^{\prime}$, and $f$ is a linear mapping of $A$ into the center of $A^{\prime}$.

Finally, there is the closely connected question of Lie derivations, that is, additive mappings, $d$, of $R$ into itself satisfying

$$
d([x, y])=[d(x), y]+[x, d(y)] \text { for all } x, y \in R .
$$

It is easy to verify that $d$ satisfies

$$
\left[d\left(x^{2}\right)-d(x) x-x d(x), x\right]=0 \text { for all } x \in R .
$$

In view of this observation we will study additive mappings satisfying (3). The relation (2) can be written in the form $\left[\theta\left(\theta^{-1}\left(y^{2}\right)\right)-y^{2}, y\right]=0$, and one observes the analogy with relation (3); using a similar approach as in the proof of Theorem 2 we will prove

Theorem 4. Let $R$ be a prime ring of characteristic different from 2 and 3 . Suppose that an additive mapping $d: R \rightarrow R$ satisfies (3). If $R$ does not satisfy $S_{4}$ then $d$ is of the form

$$
d(x)=\gamma x+\delta(x)+\zeta(x) \text { for all } x \in R,
$$

where $\gamma$ is an element in $C, \delta$ is a derivation of $R$ into its central closure, and $\zeta$ is an additive mapping of $R$ into $C$.

A Lie triple derivation is an additive mapping, $d$, of $R$ into itself satisfying

$$
\begin{array}{r}
d([[x, y], z])=[[d(x), y], z]+[[x, d(y)], z]+[[x, y], d(z)] \\
\text { for all } x, y, z \in R .
\end{array}
$$

Clearly, Lie derivations and Jordan triple derivations (cf. [4]) are the special cases of Lie triple derivations.

In [30] Miers studied Lie triple derivations of von Neumann algebras. There does not seem to be a ring theoretic analogue of Miers's result. As an application of Theorem 4 we will, however, obtain one such result.

Corollary 2. Let $R$ be a prime ring of characteristic different from 2 and 3. Let $d$ be a Lie triple derivation of $R$. If $R$ does not satisfy $S_{4}$ then $d$ is of the form $\delta+\zeta$, where $\delta$ is a derivation of $R$ into its central closure and $\zeta$ is an additive mapping of $R$ into $C$.

Adapting the proof of Theorem 3 we will show that the assumption that the characteristic of $R$ is not 3 can be removed in the case of Lie derivations.

Theorem 5. Let $R$ be a prime ring of characteristic not 2. Let $d$ be a Lie derivation of $R$. If $R$ does not satisfy $S_{4}$ then $d$ is of the form $\delta+\tau$, where $\delta$ is a derivation of $R$ into its central closure and $\tau$ is an additive mapping of $R$ into $C$ sending commutators to zero.

Theorem 5 can be compared with a result of Martindale [22]. He obtained the analogous conclusion under the assumption that $R$ is primitive containing a nontrivial idempotent. 
We close this section by listing a few more or less well-known results which will be needed in the sequel. First, by standard $P I$ theory, prime rings satisfying $S_{4}$ can be characterized in several ways:

Lemma 1. Let $R$ be a prime ring. Then the following statements are equivalent:

(i) $R$ satisfies $S_{4}$.

(ii) $R$ is commutative or $R$ embeds in $M_{2}(F)$ for $F$ a field.

(iii) $R$ is algebraic of bounded degree 2 over $C$ (i.e., for any $a \in R$ there exists a polynomial $x^{2}+\alpha_{1} x+\alpha_{2} \in C[x]$ satisfied by a).

(iv) $R$ satisfies $\left[\left[x^{2}, y\right],[x, y]\right]$.

We will make crucial use of the following lemma.

Lemma 2 [23, Theorem 1]. Suppose that elements $a, b$ in a prime ring $R$ satisfy $a x b=b x a$ for all $x \in R$. If $a \neq 0$ then $b=\lambda a$ for some $\lambda \in C$.

The next lemma is a very special case of Posner's theorem on centralizing derivations (on the other hand, a direct proof can be easily done).

Lemma 3. If a derivation $\delta$ of a noncommutative prime ring $R$ maps $R$ into its center, then $\delta=0$.

Finally, we state another well-known theorem of Posner.

Lemma 4 [34, Theorem 1]. Let $R$ be a prime ring of characteristic not 2 . If $d$ and $g$ are derivations of $R$ such that the composition $d g$ is also a derivation, then either $d=0$ or $g=0$.

\section{Proofs}

Proof of Theorem 1. There exists a biadditive mapping $B: R \times R \rightarrow R$ such that $q(x)=B(x, x)$. Since the characteristic of $R$ is not $2, C$ contains the element $\frac{1}{2}$ (i.e, the element $1+1$ is invertible). Therefore we may assume that $B$ is symmetric (i.e., $B(x, y)=B(y, x)$ for all $x, y \in R)$-otherwise replace $B$ by the mapping $(x, y) \mapsto B(x, y)+B(y, x)$.

Replacing $x$ by $x+y$ in $[B(x, x), x]=0$ we get

$$
\begin{array}{r}
{[B(x, x), y]+2[B(x, y), x]+2[B(x, y), y]+[B(y, y), x]=0} \\
\text { for all } x, y \in R .
\end{array}
$$

Take $-y$ for $y$ in (1) and compare the relation, so obtained, with (1). Since the characteristic of $R$ is not 2 it follows that

$$
[B(x, x), y]+2[B(x, y), x]=0 \text { for all } x, y \in R \text {. }
$$

A linearization of $(2)$ gives

$$
\begin{array}{r}
{[B(x, z), y]+[B(x, y), z]+[B(y, z), x]=0} \\
\text { for all } x, y, z \in R .
\end{array}
$$

Replacing $z$ by $z w$ in $(3)$ we get $[B(x, z w), y]+[B(x, y), z] w+z[B(x, y), w]$ $+[B(y, z w), x]=0$. According to (3) this relation can be written in the form

$$
\begin{aligned}
& {[B(x, z w), y]+[B(y, z w), x]} \\
& \quad=[B(x, z), y] w+[B(y, z), x] w+z[B(x, w), y] \\
& \quad+z[B(y, w), x] \text { for all } x, y, z, w \in R .
\end{aligned}
$$


Now fix $z, w \in R$ and introduce the mapping $M: R \times R \rightarrow R$ by

$$
M(x, y)=[B(x, z w), y]+z[y, B(x, w)]+[y, B(x, z)] w .
$$

On the other hand, from (4) we see that

$$
M(x, y)=[x, B(y, z w)]+z[B(y, w), x]+[B(y, z), x] w .
$$

By (5) we see that the mapping $y \mapsto M(x, y)$ is equal to the sum of compositions of inner derivations and one-sided multiplications. Analogously, (6) tells us that the same is true for the mapping $x \mapsto M(x, y)$. These two observations are the concept behind the proof of the theorem.

Comparing (5) and (6) we obtain

$$
M(x, x)=0 \text { for all } x \in R .
$$

Consider $M(y, x w)$ where $x$ and $y$ are arbitrary elements in $R$ and $w$ is the fixed element. Using (5) we obtain

$$
\begin{aligned}
M(y, x w)= & {[B(y, z w), x w]+z[x w, B(y, w)]+[x w, B(y, z)] w } \\
= & {[B(y, z w), x] w+x[B(y, z w), w]+z x[w, B(y, w)] } \\
& +z[x, B(y, w)] w+x[w, B(y, z)] w+[x, B(y, z)] w^{2} \\
= & \{[B(y, z w), x]+z[x, B(y, w)]+[x, B(y, z)] w\} w \\
& +x\{[B(y, z w), w]+z[w, B(y, w)]+[w, B(y, z)] w\} \\
& +[z, x][w, B(y, w)] .
\end{aligned}
$$

Thus

$$
\begin{array}{r}
M(y, x w)=M(y, x) w+x M(y, w)+[z, x][w, B(y, w)] \\
\text { for all } x, y \in R .
\end{array}
$$

In particular,

$$
\begin{array}{r}
M(x y, x w)=M(x y, x) w+x M(x y, w)+[z, x][w, B(x y, w)] \\
\text { for all } x, y \in R .
\end{array}
$$

In view of (9) we now consider the expression $M(x y, u)$. From (6) it follows that

$$
\begin{aligned}
M(x y, u)= & {[x y, B(u, z w)]+z[B(u, w), x y]+[B(u, z), x y] w } \\
= & x[y, B(u, z w)]+[x, B(u, z w)] y+z x[B(u, w), y] \\
& +z[B(u, w), x] y+[B(u, z), x] y w+x[B(u, z), y] w,
\end{aligned}
$$

and note that this relation can be written as

$$
\begin{aligned}
M(x y, u)= & x M(y, u)+[z, x][B(u, w), y] \\
& +M(x, u) y+[B(u, z), x][y, w] \text { for all } x, y, u \in R .
\end{aligned}
$$

In particular,

$$
\begin{aligned}
M(x y, w)= & x M(y, w)+[z, x][B(w, w), y] \\
& +M(x, w) y+[B(w, z), x][y, w] .
\end{aligned}
$$


According to (10) and (7) we have $M(x y, x)=x M(y, x)+[z, x][B(x, w), y]$ $+[B(x, z), x][y, w]$. Applying the last two relations in (9) one obtains

$$
\begin{aligned}
M(x y, x w)= & x M(y, x) w+[z, x][B(x, w), y] w \\
& +[B(x, z), x][y, w] w+x^{2} M(y, w) \\
& +x[z, x][B(w, w), y]+x M(x, w) y \\
& +x[B(w, z), x][y, w]+[z, x][w, B(x y, w)] \\
& \quad \text { for all } x, y \in R .
\end{aligned}
$$

With this, the expression $M(x y, x w)$ has been computed in the first way. We begin the computation in the second way by making use of $(10)$ :

$$
\begin{aligned}
M(x y, x w)= & x M(y, x w)+[z, x][B(x w, w), y] \\
& +M(x, x w) y+[B(x w, z), x][y, w] \\
& \text { for all } x, y \in R .
\end{aligned}
$$

By (7) and (8) we have $M(x, x w)=x M(x, w)+[z, x][w, B(x, w)]$. Applying (8) and the last relation in (12) one obtains

$$
\begin{aligned}
M(x y, x w)= & x M(y, x) w+x^{2} M(y, w)+x[z, x][w, B(y, w)] \\
& +[z, x][B(x w, w), y]+x M(x, w) y \\
& +[z, x][w, B(x, w)] y+[B(x w, z), x][y, w] \\
& \quad \text { for all } x, y \in R .
\end{aligned}
$$

Comparing (11) and (13) we arrive at

$$
\begin{aligned}
{[z, x][} & B(x, w), y] w+[B(x, z), x][y, w] w+x[z, x][B(w, w), y] \\
& +x[B(w, z), x][y, w]+[z, x][w, B(x y, w)] \\
= & x[z, x][w, B(y, w)]+[z, x][B(x w, w), y] \\
& +[z, x][w, B(x, w)] y+[B(x w, z), x][y, w]
\end{aligned}
$$

for all $x, y, z, w \in R$.

By (2) the following identities hold:

$$
\begin{aligned}
& 2[B(x, z), x][y, w] w=-[B(x, x), z][y, w] w, \\
& 2[z, x][w, B(x y, w)]=-[z, x][x y, B(w, w)], \\
& 2 x[z, x][w, B(y, w)]=-x[z, x][y, B(w, w)] .
\end{aligned}
$$

Multiply (14) by 2 and apply the above identities. The relation which we obtain can be written in the form

$$
\begin{aligned}
2[z, x] & B(x, w) y w-2[z, x] y B(x, w) w-[B(x, x), z] y w^{2} \\
& +[B(x, x), z] w y w+2 x[z, x] B(w, w) y-2 x[z, x] y B(w, w) \\
& +2 x[B(w, z), x] y w-2 x[B(w, z), x] w y-[z, x] x y B(w, w) \\
& +[z, x] B(w, w) x y \\
= & -x[z, x] y B(w, w)+x[z, x] B(w, w) y+2[z, x] B(x w, w) y \\
& -2[z, x] y B(x w, w)+2[z, x][w, B(x, w)] y \\
& +2[B(x w, z), x] y w-2[B(x w, z), x] w y .
\end{aligned}
$$


That is,

$$
\begin{aligned}
& {[z, x] y f_{1}(x, w)+\left[x^{2}, z\right] y q(w)+[z, B(x, x)] y w^{2}} \\
& \quad+f_{2}(x, z, w) y w+f_{3}(x, z, w) y=0 \text { for all } x, y, z, w \in R
\end{aligned}
$$

where

$$
\begin{aligned}
f_{1}(x, w)=2 B & (x w, w)-2 B(x, w) w, \\
f_{2}(x, z, w)= & 2[z, x] B(x, w)+[B(x, x), z] w \\
& +2 x[B(w, z), x]-2[B(x w, z), x], \\
f_{3}(x, z, w)= & x[z, x] B(w, w)-2 x[B(w, z), x] w \\
& +[z, x] B(w, w) x-2[z, x] B(x w, w) \\
& -2[z, x][w, B(x, w)]+2[B(x w, z), x] w .
\end{aligned}
$$

Replacing $y$ by $y[z, x] u$ in (15) we obtain

$$
\begin{aligned}
&-[z, x] y[z, x] u f_{1}(x, w) \\
&= {\left[x^{2}, z\right] y[z, x] u q(w)+[z, B(x, x)] y[z, x] u w^{2} } \\
&+f_{2}(x, z, w) y[z, x] u w+f_{3}(x, z, w) y[z, x] u .
\end{aligned}
$$

On the other hand, again using (15), we get

$$
\begin{gathered}
-[z, x] y[z, x] u f_{1}(x, w)=[z, x] y\left(-[z, x] u f_{1}(x, w)\right) \\
=[z, x] y\left[x^{2}, z\right] u q(w)+[z, x] y[z, B(x, x)] u w^{2} \\
+[z, x] y f_{2}(x, z, w) u w+[z, x] y f_{3}(x, z, w) u .
\end{gathered}
$$

Comparing the last two relations we arrive at

$$
\begin{aligned}
& \left(\left[x^{2}, z\right] y[z, x]-[z, x] y\left[x^{2}, z\right]\right) u q(w) \\
& \quad=g_{1}(x, y, z) u w^{2}+g_{2}(x, y, z, w) u w+g_{3}(x, y, z, w) u,
\end{aligned}
$$

where

$$
\begin{aligned}
& g_{1}(x, y, z)=[z, x] y[z, B(x, x)]-[z, B(x, x)] y[z, x], \\
& g_{2}(x, y, z, w)=[z, x] y f_{2}(x, z, w)-f_{2}(x, z, w) y[z, x], \\
& g_{3}(x, y, z, w)=[z, x] y f_{3}(x, z, w)-f_{3}(x, z, w) y[z, x] .
\end{aligned}
$$

From Lemmas 1 and 2 it follows that we may pick $x, y, z \in R$ such that the element $a=\left[x^{2}, z\right] y[x, z]-[x, z] y\left[x^{2}, z\right]$ is not zero. We introduce the element $b$ and the mappings $F$ and of $R$ by $b=g_{1}(x, y, z)$, $F(w)=g_{2}(x, y, z, w), G(w)=g_{3}(x, y, z, w)$. Observe that $F$ is an additive mapping. By (16) we have

$$
a u q(w)=b u w^{2}+F(w) u w+G(w) u \text { for all } u, w \in R .
$$

Replacing $u$ by $z a u$ in (17) we get $\operatorname{azauq}(w)=b z a u w^{2}+F(w) z a u w+$ $G(w) z a u$. On the other hand (17) yields

$$
\operatorname{azauq}(w)=\operatorname{az}(\operatorname{auq}(w))=a z b u w^{2}+a z F(w) u w+a z G(w) u
$$

Comparing the last two relations one obtains

$$
\begin{aligned}
& (b z a-a z b) u w^{2}+(F(w) z a-a z F(w)) u w \\
& \quad+(G(w) z a-a z G(w)) u=0 \text { for all } z, w, u \in R
\end{aligned}
$$


Replace $u$ by $u x$ in (18) and compare this relation by the relation which we obtain by multiplying (18) from the right by $x$. Then we get

$$
\begin{array}{r}
(b z a-a z b) u\left[w^{2}, x\right]+(F(w) z a-a z F(w)) u[w, x]=0 \\
\text { for all } x, z, w, u \in R .
\end{array}
$$

Substituting $u[w, x] y$ for $u$ in (19) we obtain

$$
\begin{aligned}
& (b z a-a z b) u[w, x] y\left[w^{2}, x\right] \\
& \quad+(F(w) z a-a z F(w)) u[w, x] y[w, x]=0 .
\end{aligned}
$$

By (19), the second term in this relation is also equal to

$$
-(b z a-a z b) u\left[w^{2}, x\right] y[w, x]
$$

Hence

$$
\begin{array}{r}
(b z a-a z b) R\left([w, x] y\left[w^{2}, x\right]-\left[w^{2}, x\right] y[w, x]\right)=0 \\
\text { for all } z, w, x, y \in R .
\end{array}
$$

As we have pointed out above, there exist $w, x, y \in R$ such that

$$
[w, x] y\left[w^{2}, x\right] \neq[w, x] y\left[w^{2}, x\right] .
$$

Therefore (20) implies that $b z a=a z b$ for all $z \in R$. Since $a \neq 0$ Lemma 2 tells us that $b=\lambda a$ for some $\lambda \in C$.

Now, (19) reduces to

$$
(F(w) z a-a z F(w)) R[w, x]=0 \text { for all } w, x, z \in R .
$$

Hence, for any $w \in R$ we have either $w \in Z$ or $F(w) z a=a z F(w)$ for all $z \in R$. The mapping $F$ is additive and so the set of all elements $w$ satisfying the last relation is an additive subgroup of $R$. But a group cannot be the union of two proper subgroups. By assumption, $R$ is noncommutative hence $F(w) z a=a z F(w)$ for all $w, z \in R$. Lemma 2 implies that for any $w \in R$ there exists $\mu(w) \in C$ such that $F(w)=\mu(w) a$. Since $F$ is additive it follows that $(\mu(w+u)-\mu(w)-\mu(u)) a=0$ for all $w, u \in R$; consequently $\mu$ is additive.

We have showed that the first two terms in (18) are equal to zero, so it follows that $G(w) z a=a z G(w)$ for all $w, z \in R$. Hence, for every $w \in R$ we have $G(w)=\nu(w) a$ for some $\nu(w)$ in $C$.

Applying the last statements in (17) we obtain

$$
a u\left(q(w)-\lambda w^{2}-\mu(w) w-\nu(w)\right)=0 \text { for all } u, w, \in R .
$$

Since $a \neq 0$ we are forced to conclude that $q(w)=\lambda w^{2}+\mu(w) w+\nu(w)$ holds for all $w \in R$. The proof of the theorem is complete.

Proof of Theorem 2. Taking $\theta^{-1}(y), y \in A^{\prime}$, for $x$ in $\left[\theta(x), \theta\left(x^{2}\right)\right]=0$, it follows that

$$
\left[y, \theta\left(\theta^{-1}(y)^{2}\right)\right]=0 \text { for all } y \in A^{\prime} .
$$

That is, the mapping $q(y)=\theta\left(\theta^{-1}(y)^{2}\right)$ is commuting. Since $q$ is a trace of a biadditive mapping $B: A^{\prime} \times A^{\prime} \rightarrow A^{\prime}, B(y, z)=\theta\left(\theta^{-1}(y) \theta^{-1}(z)\right)$, all the requirements of Theorem 1 are fulfilled. By assumption, the extended centroid of $A^{\prime}$ is equal to $F 1^{\prime}$ where $1^{\prime}$ is the unit element of $A^{\prime}$. Hence

$$
\theta\left(\theta^{-1}(y)^{2}\right)=\lambda y^{2}+\mu_{1}(y) y+\nu_{1}(y) \text { for all } y \in A^{\prime},
$$


where $\lambda \in F 1^{\prime}, \mu_{1}$ is an additive mapping from $A^{\prime}$ to $F 1^{\prime}$, and $\nu_{1}$ is a mapping from $A^{\prime}$ to $F 1^{\prime}$.

Since the mapping $\theta$ is linear, $B$ is in fact a bilinear mapping. If one glances through the proof of Theorem 1 one notices that this implies that $\mu_{1}$ is linear.

We set $\mu=\mu_{1} \theta, \nu=\nu_{1} \theta$. Thus $\mu$ and $\nu$ are mappings of $A$ into $F 1^{\prime}$ and $\mu$ is linear. Note that the relation (2) can be written in the form

$$
\theta\left(x^{2}\right)=\lambda \theta(x)^{2}+\mu(x) \theta(x)+\nu(x) \text { for all } x \in A .
$$

In order to show that $\lambda \neq 0$ we first prove that $\theta$ maps $F 1$ onto $F 1^{\prime}$. Since $\theta$ is linear it suffices to show that the element $\theta(1)$ lies in $F 1^{\prime}$. Replacing $x$ by $x+1$ in $\left[\theta\left(x^{2}\right), \theta(x)\right]=0$ we obtain

$$
\left[\theta\left(x^{2}\right)+2 \theta(x)+\theta(1), \theta(x)+\theta(1)\right]=0 .
$$

Since $\left[\theta\left(x^{2}\right), \theta(x)\right]=0$ this relation reduces to $\left[\theta\left(x^{2}+x\right), \theta(1)\right]=0$ where $x$ is an arbitrary element in $A$. Taking $x+1$ for $x$ in this relation we arrive at $2[\theta(x), \theta(1)]=0$. Since $\theta$ is onto and since the characteristic of $A^{\prime}$ is not 2 , this means that $\theta(1)$ lies in the center $F 1^{\prime}$ of $A^{\prime}$.

Suppose that $\lambda=0$. By (3) we then have $\theta\left(x^{2}\right)-\mu(x) \theta(x) \in F 1^{\prime}$ for every $x \in A$. Since $\mu$ maps into $F 1^{\prime}$ we may write $\mu(x)=g(x) 1^{\prime}$ where $g(x) \in F$; consequently $\theta\left(x^{2}-g(x) x\right) \in F 1^{\prime}$. But then $x^{2}-g(x) x \in F 1$, as we have showed. This contradicts the assumption that $A$ does not satisfy $S_{4}$ (Lemma 1). Thus $\lambda \neq 0$.

Define the mapping $\varphi: A \rightarrow A^{\prime}$ by

$$
\varphi(x)=\lambda \theta(x)+\frac{1}{2} \mu(x) .
$$

Of course, $\varphi$ is a linear mapping. Our goal is to show that $\varphi$ is a Jordan homomorphism (i.e., $\varphi\left(x^{2}\right)=\varphi(x)^{2}$ for all $x \in A$ ). According to (3) and (4) we have

$$
\begin{aligned}
\varphi\left(x^{2}\right) & =\lambda \theta\left(x^{2}\right)+\frac{1}{2} \mu\left(x^{2}\right) \\
& =\lambda^{2} \theta(x)^{2}+\lambda \mu(x) \theta(x)+\lambda \nu(x)+\frac{1}{2} \mu\left(x^{2}\right),
\end{aligned}
$$

while

$$
\varphi(x)^{2}=\left(\lambda \theta(x)+\frac{1}{2} \mu(x)\right)^{2}=\lambda^{2} \theta(x)^{2}+\lambda \mu(x) \theta(x)+\frac{1}{4} \mu(x)^{2} .
$$

Comparing these two relations we get

$$
\varphi\left(x^{2}\right)-\varphi(x)^{2} \in F 1^{\prime} \quad \text { for all } x \in A .
$$

Define the mapping $\varepsilon: A \times A \rightarrow A^{\prime}$ by

$$
\varepsilon(x, y)=\varphi(x y+y x)-\varphi(x) \varphi(y)-\varphi(y) \varphi(x) .
$$

Clearly $\varepsilon$ is a symmetric bilinear mapping. Linearizing (5) we see that $\varepsilon$ in fact maps into $F 1^{\prime}$. Of course, $\varphi$ is a Jordan homomorphism if and only if $\varepsilon(x, y)=0$ for all $x, y \in A$. have

Pick $x, y \in A$ and consider $W=\varphi(x(x y+y x)+(x y+y x) x)$. By (6) we

$$
\begin{aligned}
W= & \varphi(x) \varphi(x y+y x)+\varphi(x y+y x) \varphi(x)+\varepsilon(x, x y+y x) \\
= & \varphi(x)\{\varphi(x) \varphi(y)+\varphi(y) \varphi(x)+\varepsilon(x, y)\} \\
& +\{\varphi(x) \varphi(y)+\varphi(y) \varphi(x)+\varepsilon(x, y)\} \varphi(x)+\varepsilon(x, x y+y x) \\
= & \varphi(x)^{2} \varphi(y)+2 \varphi(x) \varphi(y) \varphi(x)+\varphi(y) \varphi(x)^{2} \\
& +2 \varepsilon(x, y) \varphi(x)+\varepsilon(x, x y+y x) .
\end{aligned}
$$


On the other hand,

$$
\begin{aligned}
W= & 2 \varphi(x y x)+\varphi\left(x^{2} y+y x^{2}\right) \\
= & 2 \varphi(x y x)+\varphi\left(x^{2}\right) \varphi(y)+\varphi(y) \varphi\left(x^{2}\right)+\varepsilon\left(x^{2}, y\right) \\
= & 2 \varphi(x y x)+\left\{\varphi(x)^{2}+\frac{1}{2} \varepsilon(x, x)\right\} \varphi(y) \\
& +\varphi(y)\left\{\varphi(x)^{2}+\frac{1}{2} \varepsilon(x, x)\right\}+\varepsilon\left(x^{2}, y\right) \\
= & 2 \varphi(x y x)+\varphi(x)^{2} \varphi(y)+\varphi(y) \varphi(x)^{2} \\
& +\varepsilon(x, x) \varphi(y)+\varepsilon\left(x^{2}, y\right) .
\end{aligned}
$$

Comparing the two expressions so obtained for $W$, we get

$$
\begin{aligned}
\varphi(x y x)= & \varphi(x) \varphi(y) \varphi(x)+\varepsilon(x, y) \varphi(x)-\frac{1}{2} \varepsilon(x, x) \varphi(y) \\
& +\frac{1}{2} \varepsilon(x, x y+y x)-\frac{1}{2} \varepsilon\left(x^{2}, y\right) .
\end{aligned}
$$

A linearization of (7) gives

$$
\begin{aligned}
\varphi(x y z+z y x)= & \varphi(x) \varphi(y) \varphi(z)+\varphi(z) \varphi(y) \varphi(x) \\
& +\varepsilon(x, y) \varphi(z)+\varepsilon(z, y) \varphi(x) \\
& -\varepsilon(x, z) \varphi(y)+\frac{1}{2} \varepsilon(x, z y+y z) \\
& +\frac{1}{2} \varepsilon(z, x y+y x)-\frac{1}{2} \varepsilon(x z+z x, y) .
\end{aligned}
$$

Consider $U=\varphi\left(x y x^{2}+x^{2} y x\right)$. Using (8) we obtain

$$
\begin{aligned}
U= & \varphi(x) \varphi(y) \varphi\left(x^{2}\right)+\varphi\left(x^{2}\right) \varphi(y) \varphi(x)+\varepsilon(x, y) \varphi\left(x^{2}\right) \\
& +\varepsilon\left(x^{2}, y\right) \varphi(x)-\varepsilon\left(x, x^{2}\right) \varphi(y)+\frac{1}{2} \varepsilon\left(x, x^{2} y+y x^{2}\right) \\
& +\frac{1}{2} \varepsilon\left(x^{2}, x y+y x\right)-\varepsilon\left(x^{3}, y\right) .
\end{aligned}
$$

Since $\varphi\left(x^{2}\right)=\varphi(x)^{2}+\frac{1}{2} \varepsilon(x, x)$ it follows that

$$
\begin{aligned}
U= & \varphi(x) \varphi(y) \varphi(x)^{2}+\varphi(x)^{2} \varphi(y) \varphi(x)+\frac{1}{2} \varepsilon(x, x) \varphi(x) \varphi(y) \\
& +\frac{1}{2} \varepsilon(x, x) \varphi(y) \varphi(x)+\varepsilon(x, y) \varphi(x)^{2}+\varepsilon\left(x^{2}, y\right) \varphi(x) \\
& -\varepsilon\left(x, x^{2}\right) \varphi(y)+\frac{1}{2} \varepsilon(x, y) \varepsilon(x, x)+\frac{1}{2} \varepsilon\left(x, x^{2} y+y x^{2}\right) \\
& +\frac{1}{2} \varepsilon\left(x^{2}, x y+y x\right)-\varepsilon\left(x^{3}, y\right) .
\end{aligned}
$$

On the other hand, applying (6) and (7) we obtain

$$
\begin{aligned}
U= & \varphi((x y x) x+x(x y x)) \\
= & \varphi(x y x) \varphi(x)+\varphi(x) \varphi(x y x)+\varepsilon(x y x, x) \\
= & \varphi(x) \varphi(y) \varphi(x)^{2}+\varphi(x)^{2} \varphi(y) \varphi(x)+2 \varepsilon(x, y) \varphi(x)^{2} \\
& -\frac{1}{2} \varepsilon(x, x) \varphi(y) \varphi(x)-\frac{1}{2} \varepsilon(x, x) \varphi(x) \varphi(y) \\
& +\varepsilon(x, x y+y x) \varphi(x)-\varepsilon\left(x^{2}, y\right) \varphi(x)+\varepsilon(x y x, x) .
\end{aligned}
$$

Comparing the two expressions so obtained for $U$, we see that

$$
\begin{aligned}
& \varepsilon(x, x) \varphi(x) \varphi(y)+\varepsilon(x, x) \varphi(y) \varphi(x)-\varepsilon(x, y) \varphi(x)^{2} \\
& -\varepsilon\left(x, x^{2}\right) \varphi(y)+\left(2 \varepsilon\left(x^{2}, y\right)-\varepsilon(x, x y+y x)\right) \varphi(x) \in F 1^{\prime}
\end{aligned}
$$

for all $x, y \in A$. 
In particular, if $x=y$, then we have

$$
\varepsilon(x, x) \varphi(x)^{2}-\varepsilon\left(x, x^{2}\right) \varphi(x) \in F 1^{\prime} \quad \text { for all } x \in A .
$$

Consequently

$$
\varepsilon(x, x)\left[\left[\varphi(x)^{2}, u\right],[\varphi(x), u]\right]=0 \text { for all } x \in A, u \in A^{\prime} .
$$

Suppose that $x \in A$ does not satisfy the condition

$$
\left[\left[\varphi(x)^{2}, u\right],[\varphi(x), u]\right]=0 \text { for all } u \in A^{\prime} .
$$

By (11) we see that in this case $\varepsilon(x, x)=0$. But then also $\varepsilon\left(x, x^{2}\right)=0$ otherwise $\varphi(x) \in F 1^{\prime}$ by (10) and so $x$ certainly satisfies (12). Therefore (9) yields

$$
-\varepsilon(x, y) \varphi(x)^{2}+\left(2 \varepsilon\left(x^{2}, y\right)-\varepsilon(x, x y+y x)\right) \varphi(x) \in F 1^{\prime} \quad \text { for all } y \in A .
$$

Since $x$ does not satisfy (12), the above relation implies that $\varepsilon(x, y)=0$ for all $y \in A$.

Thus we have proved that given $x \in A$, either $\varepsilon(x, y)=0$ for all $y \in A$ or $x$ satisfies (12). Suppose that $\varphi$ is not a Jordan homomorphism, i.e., $\varepsilon(x, y) \neq 0$ for some $x, y \in A$. Suppose further that there is $z \in A$ which does not satisfy (12). We have showed that in this case $\varepsilon(z, y)=0$. Since $\varepsilon(x, y) \neq 0$ we then also have $\varepsilon(x+\alpha z, y) \neq 0$ for every $\alpha \in F$. Hence the elements $x+\alpha z$ satisfy (12). By assumption we may choose $u \in A$ such that

$$
t=\left[\left[\varphi(z)^{2}, u\right],[\varphi(z), u]\right] \neq 0 .
$$

Since the elements $x$ and $x+\alpha z, \alpha \in F$, satisfy (12) it follows that $\alpha r+$ $\alpha^{2} s+\alpha^{3} t=0$ for all $\alpha \in F$, where

$$
\begin{aligned}
r= & {\left[\left[\varphi(x)^{2}, u\right],[\varphi(z), u]\right] } \\
& +[[\varphi(x) \varphi(z)+\varphi(z) \varphi(x), u],[\varphi(x), u]],
\end{aligned}
$$

and

$$
\begin{aligned}
s= & {[[\varphi(x) \varphi(z)+\varphi(z) \varphi(x), u],[\varphi(z), u]] } \\
& +\left[\left[\varphi(z)^{2}, u\right],[\varphi(x), u]\right] .
\end{aligned}
$$

Replacing $\alpha$ by $-\alpha$ it follows at once that $\alpha r+\alpha^{3} t=0, \alpha \in F$; that is, $r=-\alpha^{2} t$ for any nonzero $\alpha \in F$. Since $F \neq G F(3)$ this clearly contradicts the assumption that $t \neq 0$.

Thus either $\varphi$ is a Jordan homomorphism or (12) holds for all $x \in A$. Using (4) and the fact that $\lambda \neq 0$, we see that (12) can be rewritten in the form $\left[\left[\theta(x)^{2}, u\right],[\theta(x), u]\right]=0$ for all $u \in A^{\prime}$. Since $\theta$ is onto it follows from Lemma 1 that this relation cannot be fulfilled for all $x$ in $A$. Hence $\varphi$ is a Jordan homomorphism.

Assume that $\varphi(x)=0$ for some nonzero $x \in A$. By (4) we see that in this case $\theta(x) \in F 1^{\prime}$. At the beginning of the proof we have shown that this yields $x \in F 1$. But then, since $\varphi$ is linear, we must have $\varphi(1)=0$. Since $\varphi$ is a Jordan homomorphism this relation implies that

$$
2 \varphi(y)=\varphi(1 y+y 1)=\varphi(1) \varphi(y)+\varphi(y) \varphi(1)=0
$$

for all $y \in A$. Thus $\varphi=0$. From (4) it follows that $\theta$ maps $A$ into $F 1^{\prime}$. However, $\theta$ is onto, so we may conclude that $\varphi$ is one-to-one. 
Let us write $\lambda^{-1}$ as $c 1^{\prime}$ where $c \in F$. From (4) it follows that $\theta(x)=$ $c \varphi(x)+f(x)$ where $f(x)=-\frac{1}{2} c \mu(x)$. Also, $f(x)$ can be written in the form $h(x) 1^{\prime}, h(x) \in F$. We have proved that $\theta$ maps $F 1$ into $F 1^{\prime}$. In particular, $\varphi(1)=a 1^{\prime}$ for some nonzero $a \in F$. Hence the relation $\theta(x)=c \varphi(x)+h(x) 1^{\prime}$ can be written as $\theta(x)=\varphi\left(c x+h(x) a^{-1} 1\right)$. Consequently $\varphi$ is onto. A wellknown Herstein's theorem then tells us that $\varphi$ is either a homomorphism or an antihomomorphism [13]. The proof of the theorem is thereby completed.

Proof of Theorem 3. Of course, a Lie isomorphism $\theta$ satisfies $\left[\theta\left(x^{2}\right), \theta(x)\right]=0$ for all $x \in R$. First we argue as in the proof of Theorem 2. Observing that $\left[y, \theta\left(\theta^{-1}(y)^{2}\right)\right]=0$ for all $y \in R^{\prime}$ and then applying Theorem 1, one shows that

$$
\theta\left(x^{2}\right)=\lambda \theta(x)^{2}+\mu(x) \theta(x)+\nu(x) \text { for all } x \in R,
$$

where $\lambda$ is an element in the extended centroid $C^{\prime}$ of $R^{\prime}, \mu$ and $\nu$ are mappings of $R$ into $C^{\prime}$, and $\mu$ is additive.

Suppose that $\lambda=0$. By (1) we then have $\theta\left(x^{2}\right)-\mu(x) \theta(x) \in C^{\prime}$, and therefore

$$
\begin{aligned}
\theta\left(\left[\left[x^{2}, y\right],[x, y]\right]\right) & =\left[\left[\theta\left(x^{2}\right), \theta(y)\right],[\theta(x), \theta(y)]\right] \\
& =\mu(x)[[\theta(x), \theta(y)],[\theta(x), \theta(y)]] \\
& =0
\end{aligned}
$$

for all $x, y \in R$. Consequently $\left[\left[x^{2}, y\right],[x, y]\right]=0$ for all $x, y \in R$, which contradicts Lemma 1 . This means that $\lambda \neq 0$.

Define the mapping $\varphi$ of $R$ into the central closure of $R^{\prime}$ by

$$
\varphi(x)=\lambda \theta(x)+\frac{1}{2} \mu(x), \quad x \in R,
$$

and, as in the proof of Theorem 2, note that

$$
\varphi\left(x^{2}\right)-\varphi(x)^{2} \in C^{\prime} \quad \text { for all } x \in R .
$$

Linearizing (3) we obtain

$$
\varphi(x y+y x)-\varphi(x) \varphi(y)-\varphi(y) \varphi(x) \in C^{\prime} \quad \text { for all } x, y \in R .
$$

By (2) we have

$$
\begin{aligned}
\varphi([x, y]) & =\lambda \theta([x, y])+\frac{1}{2} \mu([x, y]) \\
& =\lambda[\theta(x), \theta(y)]+\frac{1}{2} \mu([x, y]) \\
& =\lambda^{-1}\left[\lambda \theta(x)+\frac{1}{2} \mu(x), \lambda \theta(y)+\frac{1}{2} \mu(y)\right]+\frac{1}{2} \mu([x, y]) \\
& =\lambda^{-1}[\varphi(x), \varphi(y)]+\frac{1}{2} \mu([x, y]) .
\end{aligned}
$$

Thus

$$
\varphi([x, y])-\lambda^{-1}[\varphi(x), \varphi(y)] \in C^{\prime} \text { for all } x, y \in R .
$$

Note that (4) and (5) yield

$$
2 \varphi(x y)-\left(1+\lambda^{-1}\right) \varphi(x) \varphi(y)-\left(1-\lambda^{-1}\right) \varphi(y) \varphi(x) \in C^{\prime} \quad \text { for all } x, y \in R .
$$

That is, the mapping

$$
\eta(x, y)=\varphi(x y)-\frac{1}{2}\left(1+\lambda^{-1}\right) \varphi(x) \varphi(y)-\frac{1}{2}\left(1-\lambda^{-1}\right) \varphi(y) \varphi(x)
$$


maps from $R \times R$ into $C^{\prime}$. Denote $\frac{1}{2}\left(1+\lambda^{-1}\right)$ by $\alpha$. Thus we have

$$
\varphi(x y)=\alpha \varphi(x) \varphi(y)+(1-\alpha) \varphi(y) \varphi(x)+\eta(x, y) .
$$

We want to show that $\eta(x, y)=0$ for all $x, y \in R$, and that either $\alpha=0$ or $\alpha=1$.

According to (6) we have

$$
\begin{aligned}
\varphi(x y z)= & \varphi(x(y z)) \\
= & \alpha \varphi(x) \varphi(y z)+(1-\alpha) \varphi(y z) \varphi(x)+\eta(x, y z) \\
= & \alpha \varphi(x)\{\alpha \varphi(y) \varphi(z)+(1-\alpha) \varphi(z) \varphi(y)+\eta(y, z)\} \\
& +(1-\alpha)\{\alpha \varphi(y) \varphi(z)+(1-\alpha) \varphi(z) \varphi(y)+\eta(y, z)\} \varphi(x) \\
& +\eta(x, y z) \\
= & \alpha^{2} \varphi(x) \varphi(y) \varphi(z)+\alpha(1-\alpha) \varphi(x) \varphi(z) \varphi(y) \\
& +\alpha(1-\alpha) \varphi(y) \varphi(z) \varphi(x)+(1-\alpha)^{2} \varphi(z) \varphi(y) \varphi(x) \\
& +\eta(y, z) \varphi(x)+\eta(x, y z) .
\end{aligned}
$$

On the other hand,

$$
\begin{aligned}
\varphi(x y z)= & \varphi((x y) z) \\
= & \alpha \varphi(x y) \varphi(z)+(1-\alpha) \varphi(z) \varphi(x y)+\eta(x y, z) \\
= & \alpha^{2} \varphi(x) \varphi(y) \varphi(z)+\alpha(1-\alpha) \varphi(y) \varphi(x) \varphi(z) \\
& +\alpha(1-\alpha) \varphi(z) \varphi(x) \varphi(y)+(1-\alpha)^{2} \varphi(z) \varphi(y) \varphi(x) \\
& +\eta(x, y) \varphi(z)+\eta(x y, z) .
\end{aligned}
$$

Comparing the two expressions thus obtained for $\varphi(x y z)$, we then get

$$
\begin{aligned}
& \alpha(1-\alpha)[\varphi(y),[\varphi(z), \varphi(x)]] \\
& \quad+\eta(y, z) \varphi(x)-\eta(x, y) \varphi(z) \in C^{\prime} \text { for all } x, y, z, \in R .
\end{aligned}
$$

Substituting $x^{2}$ for $z$ in (7) and using (3) we obtain $\eta\left(y, x^{2}\right) \varphi(x)-\eta(x, y) \varphi(x)^{2}$ $\in C^{\prime}$ for all $x, y \in R$. By (2) this relation can be written in the form

$$
\begin{array}{r}
-\lambda^{2} \eta(x, y) \theta(x)^{2}+\left(\lambda \eta\left(y, x^{2}\right)-\lambda \mu(x) \eta(x, y)\right) \theta(x) \in C^{\prime} \\
\text { for all } x, y \in R .
\end{array}
$$

Since $\theta$ is onto there exists $a \in R$ such that $\theta(a)$ is not an algebraic element of degree at most 2 over $C$ (Lemma 1). We have proved that $\lambda \neq 0$. Therefore, taking $a$ for $x$ in (8), we see that $\eta(a, y)=0$ for all $y \in R$. Thus (7) gives

$$
\alpha(1-\alpha)[\varphi(y),[\varphi(z), \varphi(a)]]+\eta(y, z) \varphi(a) \in C^{\prime} \quad \text { for all } y, z \in R \text {. }
$$

Our immediate goal is to prove that $\alpha=0$ or $\alpha=1$. Suppose this is not true. Then $\alpha(1-\alpha) \neq 0$, so it follows from (9) that $[\varphi(a),[\varphi(y),[\varphi(z), \varphi(a)]]]=0$ for all $y, z \in R$. By (2) this relation can be written as

$$
\lambda^{4}[\theta(a),[\theta(y),[\theta(z), \theta(a)]]]=0
$$

for all $y, z \in R$. Since $\lambda \neq 0$ and $\theta$ is a Lie isomorphism, it follows that $[a,[y,[z, a]]]=0$ for all $y, z \in R$. This relation can be written in the form

$$
[[a, y],[z, a]]=[[a,[z, a]], y] \text { for all } y, z \in R \text {. }
$$


The element $a$ does not lie in the center $Z$ of $R$-otherwise $\theta(a)$ would lie in the center of $R^{\prime}$, contrary to the assumption. In other words, the mapping $g(y)=[a, y]$ is a nonzero derivation of $R$. By Lemma 3 there is $z \in R$ such that $[z, a] \notin Z$. Thus the mapping $d(y)=[y,[z, a]]$ is a nonzero derivation of $R$. But from (10) we see that the composition $d g$ is also a derivation, which is impossible by Lemma 4. This contradiction tells us that either $\alpha=0$ or $\alpha=1$.

In any case, $\alpha(1-\alpha)=0$. Hence (9) yields $\eta(y, z) \varphi(a) \in C^{\prime}$ for all $y, z \in R$; that is, $\lambda \eta(y, z) \theta(a) \in C^{\prime}$, and, therefore, $\lambda \eta(y, z)[\theta(a), u]=0$ for all $y, z \in R, u \in R^{\prime}$. Since $\lambda \neq 0$ and $\theta(a) \notin Z^{\prime}$ it follows that $\eta(y, z)=0$ for all $y, z \in R$.

Suppose that $\alpha=0$. Then it follows by (6) that $\varphi$ is an antihomomorphism. Recall that $\alpha=\frac{1}{2}\left(1+\lambda^{-1}\right)$; thus $\lambda=-1$. Set $\tau(x)=\frac{1}{2} \mu(x)$. From (2) we see that $\theta(x)=-\varphi(x)+\tau(x)$. Clearly, this relation implies $\tau([x, y])=0$ for all $x, y \in R$. Analogously we see that if $\alpha=1$ then $\varphi$ is a homomorphism, $\lambda=1$, and $\theta(x)=\varphi(x)+\tau(x)$ where $\tau(x)=-\frac{1}{2} \mu(x)$; of course, $\tau$ sends commutators to zero.

Finally, let us show that $\varphi$ is one-to-one. If $\varphi(a)=0$ for some $a \in R$, then $\theta(a)$ lies in $C^{\prime}$ and so $a \in Z$. Of course, the kernel of $\varphi$ is an ideal of $R$. However, it is easy to see that a noncommutative prime ring does not contain any nonzero central ideal. The proof of the theorem is thus completed.

Proof of Corollary 1. Given $x, z \in A$, we have

$$
\left[\left[\theta\left(x^{2}\right), \theta(x)\right], \theta(z)\right]=\theta\left(\left[\left[x^{2}, x\right], z\right]\right)=0 .
$$

Since $\theta$ is onto this means that for any $x$ in $A$ the element $\left[\theta\left(x^{2}\right), \theta(x)\right]$ lies in the center of $Z^{\prime}$ of $A^{\prime}$. That is, $\left[\theta\left(\theta^{-1}(y)^{2}\right), y\right] \in Z^{\prime}$ for all $y \in A^{\prime}$. In other words, the mapping $q(y)=\theta\left(\theta^{-1}(y)^{2}\right)$ is centralizing. Of course, $q$ is a trace of a biadditive mapping. But in [7] we proved that in certain rings, in particular, prime rings of characteristic different from 2 and 3 , every centralizing trace of a biadditive mapping is commuting. Thus $\left[\theta\left(\theta^{-1}(y)^{2}\right), y\right]=0$ for all $y \in A^{\prime}$, i.e., $\left[\theta\left(x^{2}\right), \theta(x)\right]=0$ for all $x \in A$. By Theorem $2, \theta$ is of the form $\theta(x)=c \varphi(x)+f(x), x \in A$. Thus Corollary 1 will be proved by showing that $c=1$ or $c=-1$.

On the one hand we have

$$
\theta([[x, y], z])=[[\theta(x), \theta(y)], \theta(z)]=c^{3}[[\varphi(x), \varphi(y)], \varphi(z)] .
$$

On the other hand,

$$
\begin{aligned}
\theta([[x, y], z]) & =c \varphi([[x, y], z])+f([[x, y], z]) \\
& =c[[\varphi(x), \varphi(y)], \varphi(z)]+f([[x, y], z]) .
\end{aligned}
$$

Comparing the last two relations we obtain

$$
\left(c^{3}-c\right)[[\varphi(x), \varphi(y)], \varphi(z)]=f([[x, y], z]) \in Z^{\prime} .
$$

Take $x^{\prime} \in A^{\prime}$ such that $x^{\prime} \notin Z^{\prime}$. By Lemma 3 there is $y^{\prime} \in A^{\prime}$ such that $\left[x^{\prime}, y^{\prime}\right] \notin Z^{\prime}$. Hence, again according to Lemma 3 , there is $z^{\prime} \in A^{\prime}$ satisfying $\left[\left[x^{\prime}, y^{\prime}\right], z^{\prime}\right] \notin Z^{\prime}$. But then, since $\varphi$ is onto, it follows from (1) that $c^{3}=c$. Theorem 2 asserts that $c \neq 0$; thus $c=1$ or $c=-1$. The proof is complete. 
Proof of Theorem 4. By assumption, the mapping $x \mapsto d\left(x^{2}\right)-d(x) x-x d(x)$ is commuting. Obviously, this mapping is a trace of a biadditive mapping. Therefore it follows from Theorem 1 that

$$
d\left(x^{2}\right)-d(x) x-x d(x)=\lambda x^{2}+\mu(x) x+\nu(x), \quad x \in R,
$$

where $\lambda \in C, \mu, \nu$ are mappings from $R$ into $C$, and $\mu$ is additive.

We define $\delta$ by

$$
\delta(x)=d(x)+\lambda x+\frac{1}{2} \mu(x), \quad x \in R .
$$

Our intention is to show that $\delta$ is a Jordan derivation (i.e., $\delta\left(x^{2}\right)=\delta(x) x+$ $x \delta(x), x \in R)$. By (2) and (1) we have

$$
\begin{aligned}
\delta\left(x^{2}\right) & =d\left(x^{2}\right)+\lambda x^{2}+\frac{1}{2} \mu\left(x^{2}\right) \\
& =d(x) x+x d(x)+2 \lambda x^{2}+\mu(x) x+\nu(x)+\frac{1}{2} \mu\left(x^{2}\right),
\end{aligned}
$$

whereas $\delta(x) x+x \delta(x)=d(x) x+x d(x)+2 \lambda x^{2}+\mu(x) x$. Hence

$$
\delta\left(x^{2}\right)-\delta(x) x-x \delta(x) \in C \text { for all } x \in R .
$$

Linearizing (3) we see that

$$
\begin{array}{r}
\delta(x y+y x)=\delta(x) y+\delta(y) x+x \delta(y)+y \delta(x)+\rho(x, y) \\
\text { for all } x, y \in R,
\end{array}
$$

where $\rho$ is a symmetric biadditive mapping of $R \times R$ to $C$.

Consider $A=\delta(x(x y+y x)+(x y+y x) x)$. By (4) we have

$$
\begin{aligned}
A= & \delta(x)(x y+y x)+\delta(x y+y x) x \\
& +x \delta(x y+y x)+(x y+y x) \delta(x)+\rho(x, x y+y x) \\
= & 2 \delta(x) y x+2 x \delta(y) x+2 x y \delta(x)+\delta(x) x y+\delta(y) x^{2} \\
& +y \delta(x) x+x \delta(x) y+x^{2} \delta(y)+y x \delta(x) \\
& +2 \rho(x, y) x+\rho(x, x y+y x) .
\end{aligned}
$$

On the other hand,

$$
\begin{aligned}
A= & 2 \delta(x y x)+\delta\left(x^{2} y+y x^{2}\right) \\
= & 2 \delta(x y x)+\delta\left(x^{2}\right) y+\delta(y) x^{2} \\
& +x^{2} \delta(y)+y \delta\left(x^{2}\right)+\rho\left(x^{2}, y\right) \\
= & 2 \delta(x y x)+\delta(x) x y+x \delta(x) y+\delta(y) x^{2}+x^{2} \delta(y) \\
& +y \delta(x) x+y x \delta(x)+\rho(x, x) y+\rho\left(x^{2}, y\right) .
\end{aligned}
$$

Comparing the two expressions so obtained for $A$, we get

$$
\begin{aligned}
\delta(x y x)= & \delta(x) y x+x \delta(y) x+x y \delta(x)+\rho(x, y) x \\
& -\frac{1}{2} \rho(x, x) y+\frac{1}{2} \rho(x, x y+y x)-\frac{1}{2} \rho\left(x^{2}, y\right) .
\end{aligned}
$$

A linearization of (5) gives

$$
\begin{aligned}
\delta(x y z+z y x)= & \delta(x) y z+x \delta(y) z+x y \delta(z)+\delta(z) y x \\
& +z \delta(y) x+z y \delta(x)+\rho(x, y) z+\rho(z, y) x \\
& -\rho(x, z) y+\frac{1}{2} \rho(x, z y+y z) \\
& +\frac{1}{2} \rho(z, x y+y x)-\frac{1}{2} \rho(x z+z x, y) .
\end{aligned}
$$


Let $B=\delta\left(x y x^{2}+x^{2} y x\right)$. Applying (6) we obtain

$$
\begin{aligned}
B= & \delta(x) y x^{2}+x \delta(y) x^{2}+x y \delta\left(x^{2}\right)+\delta\left(x^{2}\right) y x+x^{2} \delta(y) x \\
& +x^{2} y \delta(x)+\rho(x, y) x^{2}+\rho\left(x^{2}, y\right) x-\rho\left(x, x^{2}\right) y \\
& +\frac{1}{2} \rho\left(x, x^{2} y+y x^{2}\right)+\frac{1}{2} \rho\left(x^{2}, x y+y x\right)-\rho\left(x^{3}, y\right) \\
= & \delta(x) y x^{2}+x \delta(y) x^{2}+x y \delta(x) x+x y x \delta(x)+\frac{1}{2} \rho(x, x) x y \\
& +\delta(x) x y x+x \delta(x) y x+\frac{1}{2} \rho(x, x) y x+x^{2} \delta(y) x+x^{2} y \delta(x) \\
& +\rho(x, y) x^{2}+\rho\left(x^{2}, y\right) x-\rho\left(x, x^{2}\right) y+\frac{1}{2} \rho\left(x, x^{2} y+y x^{2}\right) \\
& +\frac{1}{2} \rho\left(x^{2}, x y+y x\right)-\rho\left(x^{3}, y\right) .
\end{aligned}
$$

On the other hand, using (4) and (5) we see that

$$
\begin{aligned}
B= & \delta((x y x) x+x(x y x)) \\
= & \delta(x y x) x+\delta(x) x y x+x y x \delta(x)+x \delta(x y x)+\rho(x y x, x) \\
= & \delta(x) y x^{2}+x \delta(y) x^{2}+x y \delta(x) x+2 \rho(x, y) x^{2}-\frac{1}{2} \rho(x, x) y x \\
& +\left(\rho(x, x y+y x)-\rho\left(x^{2}, y\right)\right) x+\delta(x) x y x+x y x \delta(x) \\
& +x \delta(x) y x+x^{2} \delta(y) x+x^{2} y \delta(x)-\frac{1}{2} \rho(x, x) x y+\rho(x y x, x) .
\end{aligned}
$$

Comparing the last two relations we obtain

$$
\begin{aligned}
& \rho(x, x) x y+\rho(x, x) y x-\rho(x, y) x^{2}-\rho\left(x, x^{2}\right) y \\
& \quad+\left(2 \rho\left(x^{2}, y\right)-\rho(x, x y+y x)\right) x \in C \text { for all } x, y \in R .
\end{aligned}
$$

In particular, if $x=y$, then (7) gives

$$
\rho(x, x) x^{2}-\rho\left(x, x^{2}\right) x \in C \text { for all } x \in R .
$$

The relations (7) and (8) are similar to relations (9) and (10) in the proof of Theorem 2. We now argue as in the proof of Theorem 2. First, note that any $x \in R$ satisfies one of the following two conditions: either $\rho(x, y)=0$ for all $y \in R$ or $\left[\left[x^{2}, u\right],[x, u]\right]=0$ for all $u \in R$. Using the assumption that $R$ is of characteristic different from 2 and 3, one shows (considering the elements $x+z, x-z$, and $x+2 z)$ that one of these two conditions is satisfied by all $x$ in $R$; according to Lemma 1 we are then forced to conclude that $\rho(x, y)=0$ for all $x, y \in R$. Hence $\delta$ is a Jordan derivation.

However, we must show that $\delta$ is a derivation. In [14] Herstein proved that if $R$ is a prime ring of characteristic not 2 then every Jordan derivation of $R$ into itself is a derivation. Unfortunately, we cannot directly apply this result since $\delta$ maps $R$ into its central closure. Anyway, using the same approach as in [8], where a brief proof of Herstein's result is presented, we show that in this more general case we may also conclude that $\delta$ is a derivation. We sketch the proof of this assertion. Theorem 3 in [9] states that every Jordan derivation, $\delta$, of a 2-torsion free ring $R$ satisfies

$$
a^{b} r[a, b]+[a, b] r a^{b}=0 \text { for all } a, b, r \in R,
$$

where $a^{b}=\delta(a b)-\delta(a) b-a \delta(b)$. From the proof of this result we see that (9) also holds for Jordan derivations which map $R$ into a ring containing $R$. Now, let $R$ be prime and let $\delta$ map $R$ into its central closure. Replacing $r$ by $r[a, b] s$ in (9) we get $a^{b} r[a, b] s[a, b]+[a, b] r[a, b] s a^{b}=0$. But from 
(9) we see that this relation can be written as $-2[a, b] r a^{b} s[a, b]=0$ (cf. [16, Lemma 3.10]). Since $R$ is prime of characteristic not 2 , it follows that for any $a, b \in R$ we have either $a^{b}=0$ or $[a, b]=0$. Using the fact that a group cannot be the union of two proper subgroups it follows easily that either $a^{b}=0$ for all $a, b \in R$, i.e., $\delta$ is a derivation, or $R$ is commutative. However, by the definition of Jordan derivations we see that in the commutative case $\delta$ is trivially a derivation.

Finally, by (2) we have $d(x)=\gamma x+\delta(x)+\zeta(x)$ where $\gamma=-\lambda$ and $\zeta(x)=$ $-\frac{1}{2} \mu(x)$. The proof of the theorem is complete.

Proof of Corollary 2. For any $x, z \in R$ we have

$$
0=d\left(\left[\left[x^{2}, x\right], z\right]\right)=\left[\left[d\left(x^{2}\right), x\right], z\right]+\left[\left[x^{2}, d(x)\right], z\right] .
$$

Since $\left[x^{2}, d(x)\right]=-[d(x) x+x d(x), x]$ we thus have

$$
\left[\left[d\left(x^{2}\right)-d(x) x-x d(x), x\right], z\right]=0 \text { for all } x, z \in R .
$$

By a result in [7] quoted earlier (see the proof of Corollary 1) it follows that $\left[d\left(x^{2}\right)-d(x) x-x d(x), x\right]=0$ for all $x \in R$. Therefore Theorem 4 can be applied. Thus $d(x)=\gamma x+\delta(x)+\zeta(x)$. The corollary will be proved by showing that $\gamma=0$. Since both $d$ and $\delta$ are Lie triple derivations it follows from the above relation that $2 \gamma[[x, y], z]=\zeta([[x, y], z]) \in C^{\prime}$. If $\gamma \neq 0$, then $2 \gamma$ is invertible and so the last relation implies that $[[x, y], z] \in Z$ for all $x, y, z \in R$. Using Lemma 3 twice we see that this is impossible. Thus $\gamma=0$ and the corollary is proved.

Proof of Theorem 5. We have $0=d\left(\left[x^{2}, x\right]\right)=\left[d\left(x^{2}\right), x\right]+\left[x^{2}, d(x)\right]$, and note that this relation can be written in the form

$$
\left[d\left(x^{2}\right)-d(x) x-x d(x), x\right]=0 \text { for all } x \in R .
$$

As in the proof of Theorem 4 we see that (1) implies that there exist $\lambda \in C$, an additive mapping $\mu: R \rightarrow C$, and a mapping $\nu: R \rightarrow C$ such that

$$
d\left(x^{2}\right)-d(x) x-x d(x)=\lambda x^{2}+\mu(x) x+\nu(x) \text { for all } x \in R .
$$

Define the mapping $\delta$ of $R$ into the central closure of $R$ by

$$
\delta(x)=d(x)+\frac{1}{2} \mu(x), \quad x \in R .
$$

We want to show that $\delta$ is a derivation. (In the proof of Theorem 4 we defined the derivation $\delta$ by $\delta(x)=d(x)+\lambda x+\frac{1}{2} \mu(x)$-the reason for this distinction is that the element $\lambda$ is in fact equal to zero, as we shall see.)

By (2) and (3) it follows that

$$
\begin{aligned}
\delta\left(x^{2}\right) & =d\left(x^{2}\right)+\frac{1}{2} \mu\left(x^{2}\right) \\
& =d(x) x+x d(x)+\lambda x^{2}+\mu(x) x+\nu(x)+\frac{1}{2} \mu\left(x^{2}\right) \\
& =\delta(x) x+x \delta(x)+\lambda x^{2}+\nu(x)+\frac{1}{2} \mu\left(x^{2}\right) .
\end{aligned}
$$

Thus $\delta\left(x^{2}\right)-\delta(x) x-x \delta(x)-\lambda x^{2} \in C$ for every $x$ in $R$. Linearizing this relation we obtain

$$
\begin{gathered}
\delta(x y+y x)-\delta(x) y-\delta(y) x-x \delta(y)-y \delta(x) \\
-\lambda(x y+y x) \in C \text { for all } x, y \in R
\end{gathered}
$$


Next we have

$$
\begin{aligned}
\delta([x, y]) & =d([x, y])+\frac{1}{2} \mu([x, y]) \\
& =[d(x), y]+[x, d(y)]+\frac{1}{2} \mu([x, y]) \\
& =[\delta(x), y]+[x, \delta(y)]+\frac{1}{2} \mu([x, y])
\end{aligned}
$$

Thus

$$
\delta([x, y])-[\delta(x), y]-[x, \delta(y)] \in C \quad \text { for all } x, y \in R .
$$

Comparing (4) and (5) one obtains

$$
2 \delta(x y)-2 \delta(x) y-2 x \delta(y)-\lambda(x y+y x) \in C \quad \text { for all } x, y \in R,
$$

and therefore

$$
\delta(x y)=\delta(x) y+x \delta(y)+\beta(x y+y x)+\omega(x, y),
$$

where $\beta=\frac{1}{2} \lambda$ and $\omega$ is a biadditive mapping from $R \times R$ to $C$. In order to prove that $\delta$ is a derivation we will show that $\beta=0$ and $\omega=0$. that

We will compute $\delta(x y z)$ in two ways. On the one hand, using (6) we see

$$
\begin{aligned}
\delta(x y z)= & \delta(x(y z)) \\
= & \delta(x) y z+x \delta(y z)+\beta(x(y z)+(y z) x)+\omega(x, y z) \\
= & \delta(x) y z+x \delta(y) z+x y \delta(z)+\beta x(y z+z y) \\
& +\omega(y, z) x+\beta(x y z+y z x)+\omega(x, y z) .
\end{aligned}
$$

On the other hand,

$$
\begin{aligned}
\delta(x y z)= & \delta((x y) z) \\
= & \delta(x y) z+x y \delta(z)+\beta((x y) z+z(x y))+\omega(x y, z) \\
= & \delta(x) y z+x \delta(y) z+\beta(x y+y x) z+\omega(x, y) z \\
& +x y \delta(z)+\beta(x y z+z x y)+\omega(x y, z) .
\end{aligned}
$$

Comparing we get

$$
\beta[y,[z, x]]+\omega(y, z) x-\omega(x, y) z \in C \text { for all } x, y, z \in R .
$$

This relation is analogous to the relation (7) in the proof of Theorem 3 . Henceforth we argue as in the proof of Theorem 3, therefore we only sketch the rest of the proof. Taking $x^{2}$ for $z$ in (7) we find there exists $a \in R$, not algebraic over $C$ of degree at most 2 , such that $\omega(a, y)=0$ for all $y \in R$. Substituting $a$ for $x$ in (7) and applying Lemma 4, then one shows that $\beta=0$. Hence it follows easily that $\omega(y, z)=0$ for all $y, z \in R$. Consequently $\delta$ is a derivation.

Finally, define $\tau$ by $\tau(x)=-\frac{1}{2} \mu(x)$. Thus $d=\delta+\tau$ by (3). It is obvious that this relation implies that $\tau$ sends commutators into zero. The proof of the theorem is thereby completed.

\section{ACKNOWLEDGMENT}

I am indebted to my supervisor, Dr. J. Vukman, for his advice and encouragement. I would also like to thank the referee for his valuable suggestions. 


\section{REFERENCES}

1. L. B. Beasley, Linear transformations on matrices: The invariance of commuting pairs of matrices, Linear and Multilinear Algebra 6 (1978), 179-183.

2. H. E. Bell and W. S. Martindale, Centralizing mappings of semiprime rings, Canad. Math. Bull. 30 (1987), 92-101.

3. - Semiderivations and commutativity in prime rings, Canad. Math. Bull. 31 (1988), 500-508.

4. M. Brešar, Jordan mappings of semiprime rings, J. Algebra 127 (1989), 218-228.

5. $\ldots$, Centralizing mappings on von Neumann algebras, Proc. Amer. Math. Soc. 111 (1991), 501-510.

6. __ Centralizing mappings and derivations in prime rings, J. Algebra (to appear).

7. _ On a generalization of the notion of centralizing mappings, Proc. Amer. Math. Soc. 114 (1992), 641-649.

8. M. Brešar and J. Vukman, Jordan derivations on prime rings, Bull. Austral. Math. Soc. 37 (1988), 321-322.

9. __ On some additive mappings in rings with involution, Aequationes Math. 38 (1989), 178-185.

10. __ On left derivations and related mappings, Proc. Amer. Math. Soc. 110 (1990), 7-16.

11. G. H. Chan and M. H. Lin, Linear transformations on symmetric matrices that preserve commutativity, Linear Algebra Appl. 47 (1982), 11-22.

12. M. D. Choi, A. A. Jafarian, and H. Radjavi, Linear maps preserving commutativity, Linear Algebra Appl. 87 (1987), 227-242.

13. I. N. Herstein, Jordan homomorphisms, Trans. Amer. Math. Soc. 81 (1956), 331-351.

14. __ Jordan derivations of prime rings, Proc. Amer. Math. Soc. 8 (1957), 1104-1110.

15. __ On a type of Jordan mappings, An Acad. Bras. Cienc. 39 (1967), 357-360.

16. __ Topics in ring theory, Univ. of Chicago Press, Chicago, Ill., 1969.

17. __ Rings with involution, Univ. of Chicago Press, Chicago, Ill., 1976.

18. L. Hua, A theorem on matrices over an s-field and its applications, J. Chinese Math. Soc. (N.S.) 1 (1951), 110-163.

19. N. Jacobson and C. E. Rickart, Jordan homomorphisms of rings, Trans. Amer. Math. Soc. 69 (1950), 479-502.

20. C. Lanski, Differential identities, Lie ideals, and Posner's theorems, Pacific J. Math. 134 (1988), 275-297.

21. W. S. Martindale, Lie isomorphisms of primitive rings, Proc. Amer. Math. Soc. 14 (1963), 909-916.

22. __ Lie derivations of primitive rings, Michigan J. Math. 11 (1964), 183-187.

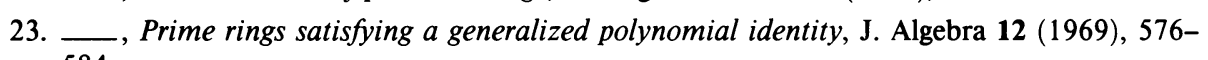
584.

24. __ Lie isomorphisms of simple rings, J. London Math. Soc. 44 (1969), 213-221.

25. __ Lie isomorphisms of prime rings, Trans. Amer. Math. Soc. 142 (1969), 437-455.

26. __ Lie and Jordan mappings in associative rings, Proc. Ohio Univ. Conf. on Ring Theory, May, 1976, Marcel Dekker.

27. M. Mathieu, Rings of quotients of ultraprime Banach algebras. With applications to elementary operators, Proc. Centre Math. Anal. Austral. Nat. Univ. 21 (1989), 297-317.

28. C. R. Miers, Lie isomorphisms of factors, Trans. Amer. Math. Soc. 147 (1970), 55-63.

29. _L Lie *-triple homomorphisms into von Neumann algebras, Proc. Amer. Math. Soc. 58 (1976), 169-172.

30. $\_$, Lie triple derivations of von Neumann algebras, Proc. Amer. Math. Soc. 71 (1978), 57-61.

31. _ Commutativity preserving maps of factors, Canad. J. Math. 40 (1988), 248-256. 
32. M. Omladič, On operators preserving commutativity, J. Funct. Anal. 66 (1986), 105-122.

33. S. Pierce and W. Watkins, Invariants of linear maps on matrix algebras, Linear and Multilinear Algebra 6 (1978), 185-200.

34. E. Posner, Derivations in prime rings, Proc. Amer. Math. Soc. 8 (1957), 1093-1100.

35. H. Radjavi, Commutativity preserving operators on symmetric matrices, Linear Algebra Appl. 61 (1984), 219-224.

36. J. Vukman, Commuting and centralizing mappings in prime rings, Proc. Amer. Math. Soc. 109 (1990), 47-52.

37. W. Watkins, Linear maps that preserve commuting pairs of matrices, Linear Algebra Appl. 14 (1976), 29-35.

Department of MAthematics, University OF MARibor, PF, Koroška 160, 62000 Maribor, SLOVENIA 